\title{
刈割干扰和养分添加条件下Epichloë内生真菌感染 对羽茅所在群落多样性和生产力的影响
}

\author{
吴 曼 李娟娟 刘金铭 任安芝* 高玉荣
}

南开大学生命科学学院, 天津 300071

摘 要 Epichloë内生真菌感染能够影响宿主植物的种内和种间竞争力, 但目前关于内生真菌感染对宿主植物所在群落多样 性和生产力影响的研究较少。该研究以感染( $\mathrm{E}+$ )和不感染( $\mathrm{E}-$-)内生真菌的羽茅(Achnatherum sibiricum)及其原生境中常见的5 种植物构建人工群落, 探究在不同养分水平和刚割条件下内生真菌对宿主植物群落的影响。结果表明: 内生真菌对宿主植物 群落多样性的影响同刈割与否有关。在无刚割条件下, 内生真菌感染对宿主群落多样性无显著影响; 在刈割条件下, 内生真 菌感染显著增加了宿主群落的多样性, 其原因在于内生真菌感染显著增加了群落中多度较小的冰草(Agropyron cristatum)和 大针茅(Stipa grandis)等的多度, 而显著降低了优势种羊草(Leymus chinensis)的多度。内生真菌感染对群落生产力未见显著影 响。研究发现养分添加对内生真菌作用的影响只出现在宿主植物水平, 而对宿主植物所在群落未产生显著影响, 内生真菌对 宿主羽茅的促进作用只出现在养分添加条件下。

关键词 内生真菌; 羽茅; 群落多样性; 生产力; 多度

吴曼, 李娟娟, 刘金铭, 任安芝, 高玉葆 (2019). 刈割干扰和养分添加条件下Epichloë内生真菌感染对羽茅所在群落多样性和生产力的影响. 植物生态 学报, 43, 85-93. DOI: 10.17521/cjpe.2018.0242

\section{Effects of Epichlö̈ infection on the diversity and productivity of Achnatherum sibiricum community under various nutrient and mowing conditions}

WU Man, LI Juan-Juan, LIU Jin-Ming, REN An-Zhi*, and GAO Yu-Bao

College of Life Sciences, Nankai University, Tianjin 300071, China

\begin{abstract}
Aims Epichloë infection can affect the intraspecific and interspecific competitive ability of the host plants. However, few studies have been reported on the effects of endophyte infection on the diversity and productivity of host communities.

Methods We used endophyte-infected (E+) and uninfected (E-) Achnatherum sibiricum, plus five other common species in its native community to construct a microcosm plant community to investigate the community-level consequences of endophyte interactions under different nutrient and mowing treatments.

Important findings Community-level consequences of endophyte infection depend on the mowing treatment. Endophyte infection did not alter plant community diversity under non-mowing conditions. Under mowing conditions, however, endophyte infection significantly increased the diversity by increasing the abundance of lower abundance species such as Agropyron cristatum and Stipa grandis, while decreasing the abundance of dominant species such as Leymus chinensis. No significant endophyte effect on the productivity of plant community was found. With respect to nutrient treatments, we found that nutrient addition had no significant effect either on the diversity or productivity of the plant community. The positive effect of endophyte on A. sibiricum occurred only under nutrient addition treatment in the host species level.
\end{abstract}

Key words fungal endophyte; Achnatherum sibiricum; community diversity; productivity; abundance

Wu M, Li JJ, Liu JM, Ren AZ, Gao YB (2019). Effects of Epichloë infection on the diversity and productivity of Achnatherum sibiricum community under various nutrient and mowing conditions. Chinese Journal of Plant Ecology, 43, 85-93. DOI: $10.17521 /$ cjpe.2018.0242

收稿日期Received: 2018-10-02 接受日期Accepted: 2019-01-11

基金项目：国家重点研发计划(2016YFC0500702)和国家自然科学基金项目(31570433)。Supported by the National Key R\&D Program of China (2016YFC0500702), and the National Natural Science Foundation of China (31570433).

* 通信作者Corresponding author (renanzhi@nankai.edu.cn) 
内生真菌(fungal endophyte)是指在植物中度过 大部分或全部的生命周期但不使宿主植物表现明显 病症的一类真菌(Arnold et al., 2000)。目前已知的感 染禾本科植物的内生真菌共有 7 个属, 分别是 Atkinsonella、Balansia、Balansiopsis、Echinodothis、 Epichloë、Myriogenospora和ParEpichloë (White \& Reddy, 1998), 其中研究较为深入的是与冷季型禾 草共生的子囊菌纲(Ascomycetes)麦角菌科(Clavicipitaceae)的Epichloë内生真菌(其无性型曾被命名 为Neotyphodium)。宿主植物研究最多的是人工禾草 黑麦草(Lolium perenne) 和苇状羊茅(Festuca arundinacea), 二者分别与内生真菌Epichloë festucae var. lolii和Epichloë coenophiala构成共生关系(Cheplick, 2004; Malinouski \& Belesky, 2006; Leuchtmann et al., 2014)。已有研究表明: 内生真菌不仅可以促进宿主 植物的生长发育(Clay, 1987), 而且能够提高宿主植 物对干旱(Elmi \& West, 1995; Oberhofer et al., 2014)、食草动物取食(Rudgers \& Clay, 2008; Schardl et al., 2012)、病原菌危害(Christensen, 1996; Xia et al., 2015)等非生物和生物胁迫的抗性。

越来越多的研究表明, 内生真菌对宿主的影响 与外界养分供给密切相关。据Saikkonen等(2006)统 计, 目前关于内生真菌对宿主植物生长有促进作用 的报道多数是在高养分条件下, 养分供给不足时的 影响可能从有利变为中性甚至不利(Marks \& Clay, 2007)。如Cheplick等(1989)在对黑麦草和苇状羊茅 幼苗的研究中发现: 在高氮条件下, 内生真菌感染 显著促进了二者的营养生长, 而在低氮条件下, 内 生真菌感染对黑麦草幼苗无显著影响, 却显著抑制 了苇状羊茅幼苗的生长。Arachevaleta等(1989)在对 苇状羊茅成熟植株的研究中发现: 在低氮条件下, 染菌与不染菌植株之间的生物量无显著差异, 而在 高氮条件下, 染菌植株的生物量显著高于不染菌植 株。Ren 等(2009)在对黑麦草成熟植株的研究中也 发现: 内生真菌只在高氮条件下显著增加宿主植物 的生物量。当然, 也有研究指出只有在低氮(Louis \& Faeth, 1997; Schulthess \& Faeth, 1998; Lewis, 2004) 或低磷(Malinowski et al., 1999; 周芳等, 2003)条件 下内生真菌感染才会促进宿主植物的生长。

内生真菌感染也能够影响宿主植物的竞争力。 以往的研究表明内生真菌感染不仅可以提高宿主植 物草甸羊茅(Festuca pratensis)、苇状羊茅、密从雀
麦(Bromus benekeni)的种内竞争能力(Marks et al., 1991; Malinowski et al., 1997; Brem \& Leuchtmann, 2002), 而且也能够提高苇状羊茅、紫羊茅(Festuca rubra)的种间竞争能力(Marks et al., 1991; Rudgers \& Orr, 2009; Vázquez-de-Aldana et al., 2013)。当然, 内生真菌对宿主植物的竞争能力也存在负效应或无 影响(Faeth et al., 2004; Moraes et al., 2014)。早在30 多年前Janos (1980) 就提出共生型真菌对驱动植物 群落演替有着重要作用, 但是目前内生真菌对群落 结构和功能影响的研究很少(Clay \& Holah, 1999; Afkhami \& Strauss, 2016)。

羽茅(Achnatherum sibiricum) 作为一种疏丛型 禾草, 是在羊草(Leymus chinensis)群落和大针茅群 落中常见的伴生种。与羽茅同为芨苃草属(Achnatherum) 的另外两个种, 即分布于我国天然草原的醉 马草(Achnatherum inebrians)和分布于美洲大陆的 Achnatherum robustum均感染内生真菌, 且均能够 产生使草食家畜中毒的生物碱(李学森等, 1998; Jones et al., 2000; Li et al., 2004), 而羽茅则不同, Zhang等(2009)发现, 在正常生长条件下, 羽茅在同 一地理种群感染有两种不同种类的优势菌株 (Epichloë gansuensis和Epichloë sibirica), 且均没有 检出生物碱, 刈割处理后, 少数染菌羽茅样品中检 出波胺, 但含量极低, 不足以对食草动物产生毒性 (Qin et al., 2016), 因此羽茅是研究内生真菌和天然 禾草共生关系的理想材料。考虑到刚割是内蒙古草 地一种最普遍的利用方式, 本研究通过构建包含内 生真菌感染 $(\mathrm{E}+)$ 和不感染 $(\mathrm{E}-)$ 羽茅的人工群落, 探 究在不同养分水平和刚割干扰处理下, 内生真菌感 染对宿主所在群落的影响, 我们提出以下 3 个科学 问题: (1)内生真菌感染是否会影响宿主所在群落的 物种多样性? (2)内生真菌感染是否会影响宿主所 在群落的生产力? (3)内生真菌对宿主群落的影响 是否与养分处理和刚割干扰有关?

\section{1 材料和方法}

\section{1 实验材料}

实验所用的植物种子和土壤均采集自中国科学 院内蒙古草原生态系统定位研究站(IMGERS)的羊 草样地 $\left(43.63^{\circ} \mathrm{N}, 116.72^{\circ} \mathrm{E}\right)$, 实验处理前测得土壤 氮、磷浓度分别为 $0.17 \%$ 和 $0.028 \%$ 。植物材料包括 内生真菌感染 $(E+)$ 和不感染 $(E-)$ 的羽茅 $\left(C_{3}\right.$ 禾草 $)$, 
羊草 $\left(\mathrm{C}_{3}\right.$ 禾草)、冰草(Agropyron cristatum $)\left(\mathrm{C}_{3}\right.$ 禾草)、 大针茅(Stipa grandis $)\left(\mathrm{C}_{3}\right.$ 禾草)、冷蒿(Artemisia frigida)(杂类草) 和糙隐子草 (Cleistogenes squar$r o s a)\left(\mathrm{C}_{4}\right.$ 禾草)。作者所在实验室通过连续多年的调 查发现，内蒙古中东部草原区的羽茅具有很高的内 生真菌侵染率, 经常接近 100\% (Wei et al., 2006)。因 此直接从野外采集 $\mathrm{E}+$ 羽茅种子, 然后, 参考李夏等 (2010)的方法，取一部分 $\mathrm{E}+$ 种子，放入烘箱，在60 ${ }^{\circ} \mathrm{C}$ 下烘干一个月得到 $\mathrm{E}-$ 羽茅种子。

根据E+和E-羽茅、羊草、冰草、大针茅、冷蒿、 糙隐子草的种子出苗时间, 先后将种子种植于装满 蛭石的苗盘中, 待幼苗的第二片叶完全展开后, 参 考Latch和Christensen (1985)的苯胺兰染色法再次确 定羽茅的内生真菌感染情况, 之后选择长势一致的 幼苗移栽至直径 $27 \mathrm{~cm}$ 高、 $20 \mathrm{~cm}$ 的花盆中, 每盆装 $6 \mathrm{~kg}$ 土壤。羽茅每盆种植 5 株, 其他 5 种植物每盆种植 2 株, 每盆一共种植 15 株植物, 所有幼苗均随机种植, 种植前土壤过 $2 \mathrm{~mm}$ 的篎子, 篮去根和石头, 定植后 的前两个星期进行补苗。实验在南开大学生命科学 学院网室中进行。

\section{2 实验设计}

本实验采用的是四因素随机区组实验设计：第 1 个因素是羽茅的内生真菌感染情况, 包括 $\mathrm{E}+$ 和 $\mathrm{E}-$ 2 个水平; 第 2 个因素是刈割处理, 包括刈割 $(\mathrm{M}+)$ 和 不㺫割(M-) 2 个水平; 第3个因素是氮处理, 包括供 氮 $(\mathrm{N}+)$ 和不供氮 $(\mathrm{N}-) 2$ 个水平; 第 4 个因素是磷处理, 包括供磷 $(\mathrm{P}+)$ 和不供磷 $(\mathrm{P}-) 2$ 个水平, 共 16 个处理, 每个处理 5 次重复, 共 80 盆, 实验持续 21 周。

氮、磷添加量参考已有的研究报道(Lan \& Bai, 2012; Yang et al., 2014, 2015), 对于供氮组, 每周施 加 $39.2 \mathrm{mmol} \cdot \mathrm{L}^{-1} \mathrm{NH}_{4} \mathrm{NO}_{3}$ 一次, 每次 $10 \mathrm{~mL}$, 相当于 $10 \mathrm{~g} \cdot \mathrm{m}^{-2} \cdot \mathrm{a}^{-1}$; 对于供磷组, 每周施加 $35.4 \mathrm{mmol} \cdot \mathrm{L}^{-1}$ $\mathrm{KH}_{2} \mathrm{PO}_{4}$ 一次, 每次 $10 \mathrm{~mL}$, 相当于 $10 \mathrm{~g} \cdot \mathrm{m}^{-2} \cdot \mathrm{a}^{-1}$ 。对照 组添加等量的水。刚割处理在幼苗定植后的第 8 周和 第16周分别进行, 留茬高度为 $5 \mathrm{~cm}$ 。

\section{3 指标测定}

种植 21 周后将全部植株收获, 置于 $80{ }^{\circ} \mathrm{C}$ 烘箱 烘干至恒质量后称质量, 并将两次刈割所得的生物 量合并, 即得总生物量。

植物叶片和土壤总磷浓度使用钼锑抗比色法 (鲍士旦, 2000)测定。

本实验起始所种的物种丰富度相同, 所以使用
的是最后一次收获的地上生物量来计算 ShannonWiener多样性指数 $\left(H^{\prime}\right)$ (Collins \& Foster, 2009):

$$
H^{\prime}=-\sum_{i=1}^{N}\left(\frac{b_{i}}{\sum_{i=1}^{N} b_{i}} \times 100\right) \times \ln \frac{b_{i}}{\sum_{i=1}^{N} b_{i}} \times 100
$$

式中, $N$ 为群落中物种的总数, $b_{i}$ 为第 $i$ 种物种生物量; 物种多度是物种 $i$ 的生物量占总生物量的百分比 (Yang et al., 2015)。

\section{4 数据的统计分析}

将所得的数据输入Excel 2007进行整理、作图, 使用SPSS 20.0进行数据统计分析。利用SPSS 20.0 对数据进行正态性检验, 对不符合正态分布的数据 进行对数转换使之符合方差分析的要求。首先在不 同刚割条件下对内生真菌感染和氮、磷添加对群落 多样性和多度影响进行三因素方差分析, 再利用多 因素方差分析评估内生真菌、氮添加、磷添加、刈 割处理以及它们之间的交互作用对群落生产力、优 势种多度、土壤磷和羽茅磷浓度的影响, 用最小显 著极差 $(L D S)$ 法进行不同处理之间的显著性检验 $(\alpha=0.05)$ 。

\section{2 结果}

\section{1 内生真菌感染对群落多样性和生产力的影响}

内生真菌对宿主植物群落多样性的影响与刈割 与否有关, 在无刚割条件下, 内生真菌感染对群落 的多样性无显著影响; 在刘割条件下, 内生真菌感 染显著增加了群落的多样性 $(p=0.002$; 图1; 附录 $\mathrm{I})$ 。

群落生产力受内生真菌感染和氮添加处理交互 作用的影响 $(p=0.003)$, 氮添加显著增加了 $\mathrm{E}-$ 羽茅

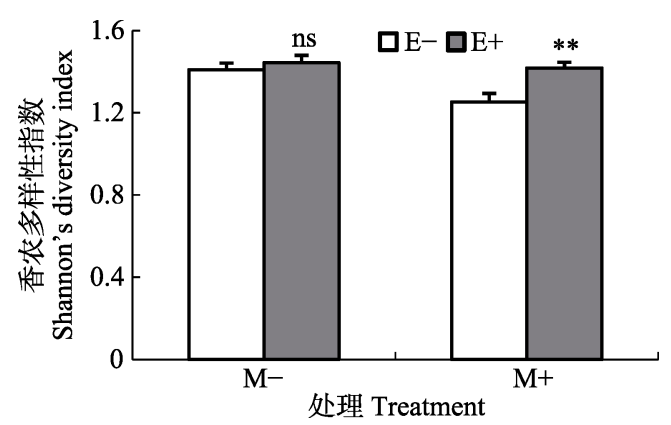

图 1 在无刈割处理 $(\mathrm{M}-)$ 和刈割处理 $(\mathrm{M}+)$ 条件下内生真菌 感染对宿主群落多样性的影响(平均值+标准误差, $n=5$ )。 $\mathrm{E}+$, 内生真菌感染; E- , 内生真菌不感染。**, $p<0.01 ; \mathrm{ns}, p>0.05$ 。 Fig. 1 Effects of endophyte infection on Shannon's diversity index of host plant communities under no-mowing $(\mathrm{M}-)$ and mowing $(\mathrm{M}+)$ conditions (mean $+S E, n=5$ ). E+, endophyteinfected; E-, endophyte-uninfected. ${ }^{* *}, p<0.01$; ns, $p>0.05$. 
所在群落的生产力, 但对 $\mathrm{E}+$ 羽茅所在群落的生产力 无显著影响; 不添加氮处理中, 内生真菌感染有增 加宿主群落生产力的趋势, 氮添加处理后内生真菌 感染的有益趋势消失(图2; 附录II)。

\section{2 内生真菌感染对群落物种多度的影响}

在无刈割处理下, 群落中羽茅和冷蒿的多度最 高, 羊草的多度次之; 在刈割处理下, 羊草的多度 升至最高, 而羽茅、冷蒿等的多度均有不同程度地 降低(图3)。

相比于养分添加, 物种多度受内生真菌感染的 影响较大, 无刈割处理时, 内生真菌感染显著降低 了羊草的多度 $(p=0.028)$ 。刏割处理后, 内生真菌不 仅显著降低了羊草的多度 $(p=0.007)$, 而且显著增 加了群落中羽茅、冰草和大针茅的多度 $(p<0.001$, $p=0.002, p<0.001)$ 。无论是否刈割, 内生真菌对 $\mathrm{C}_{4}$ 植物粘隐子草和非固氮植物冷蒿的多度均无显著影 响(图3; 附录III)。

\section{3 内生真菌感染对群落优势种多度的影响}

对群落主要优势种羊草、羽茅和冷蒿的多度进 一步分析发现, 羊草的多度主要受内生真菌感染和 刏割的影响, E+羽茅群落中的羊草多度显著小于 $\mathrm{E}-$ 羽茅群落 $(p<0.001$; 图4A), 同时刈割显著增加了 羊草的多度 $(p=0.001)$ 。

羽茅多度除受内生真菌影响 $(p<0.001)$ 外, 还 受到内生真菌分别和氮添加、磷添加以及刈割交互 作用的显著影响 $(p=0.043, p=0.005, p=0.016)$, 具

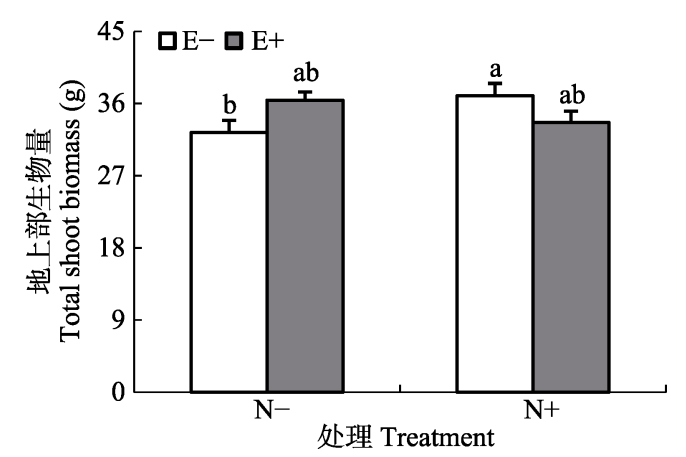

图2 内生真菌感染和氮添加对宿主群落地上生物量的影响 (平均值+标准误差, $n=5$ )。 $\mathrm{E}+$, 内生真菌感染; $\mathrm{E}-$, 无内生真 菌感染; $\mathrm{N}-$, 无氮添加; $\mathrm{N}+$, 氮添加。不同小写字母表示E+、 $\mathrm{E}-$ 植物群落间差异显著 $(p<0.05)$ 。

Fig. 2 Effects of endophyte infection and nitrogen addition on the aboveground shoot biomass of host plant communities (mean $+S E, n=5$ ). $\mathrm{E}+$, endophyte-infected; $\mathrm{E}-$, endophyteuninfected; $\mathrm{N}-$, no-nitrogen addition; $\mathrm{N}+$, nitrogen addition. Different lowercase letters mean significant difference between $\mathrm{E}+$ and $\mathrm{E}-$ plants community $(p<0.05)$.

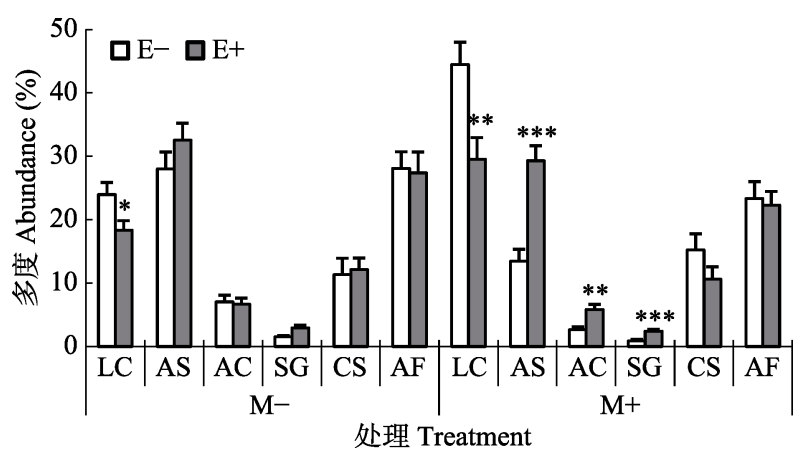

图3 在无刈割处理(M-)和刈割处理 $(\mathrm{M}+)$ 条件下内生真菌 感染对物种多度的影响(平均值+标准误差, $n=5$ )。 $\mathrm{E}+$, 内生 真菌感染; E-, 无内生真菌感染。 $\mathrm{AC}$, 冰草; $\mathrm{AF}$, 冷蒿; $\mathrm{AS}$, 羽茅; CS, 粘隐子草; LC, 羊草; SG，大针茅。星号表示E+、 $\mathrm{E}-$ 植物群落间差异显著, ${ }^{*}, p<0.05 ; * *, p<0.01$; ***, $p<$ 0.001 。

Fig. 3 Effects of endophyte infection on the species abundance under no-mowing $(\mathrm{M}-)$ and mowing $(\mathrm{M}+)$ conditions $($ mean $+S E, n=5) . \mathrm{E}+$, endophyte-infected; E-, endophyteuninfected. AC, Agropyron cristatum; AF, Artemisia frigida; AS, Achnatherum sibiricum; CS, Cleistogenes squarrosa; LC, Leymus chinensis; SG, Stipa grandis. The asterisk denotes significant difference between $\mathrm{E}+$ and $\mathrm{E}-$ plants, $*, p<0.05$; **, $p$ $<0.01 ; * * *, p<0.001$.

体表现为当分别添加氮、磷或刈割处理时，内生真 菌感染显著增加了羽茅的多度(图4B-4D); 而在无 养分添加和无刈割处理中, 内生真菌对羽茅的多度 无显著影响。磷添加和刈割作用均显著降低E-羽茅 的多度, 但对E+羽茅无显著影响(图4C、4D)。对于 冷蒿, 无论是内生真菌、养分添加还是刈割均对其 多度无显著影响(附录IV)。

\section{4 内生真菌感染对土壤和优势种磷浓度的影响}

对土壤磷浓度的测定发现, 土壤磷浓度除了受 磷添加显著影响外, 还受到内生真菌感染、氮添加、 磷添加和刈割处理四者交互作用的显著影响 $(p<$ $0.001, p=0.014)$, 磷添加显著增加了土壤的磷浓度 (图5A)。羽茅的磷浓度受到内生真菌感染和磷添加 交互作用的边缘显著影响 $(p=0.058)$, 在无磷添加 条件下, 内生真菌感染对羽茅磷浓度无明显影响; 在磷添加条件下, 内生真菌感染使羽茅磷浓度增加 了 45.7\% (图5B)。对羊草和冷蒿而言, 磷添加使 $\mathrm{E}-$ 羽茅群落中羊草和冷蒿磷浓度分别增加了 $22 \%$ 和 $21 \%$, 使 $\mathrm{E}+$ 羽茅群落中羊草和冷蒿磷浓度分别增加 了 $11 \%$ 和 $13 \%$ (图5C、5D; 附录 $\mathrm{V}$ )。

\section{3 讨论}

内生真菌感染能够影响宿主植物的生长, 改变 宿主苇状羊茅和黑麦草所在群落的种间关系(Marks 

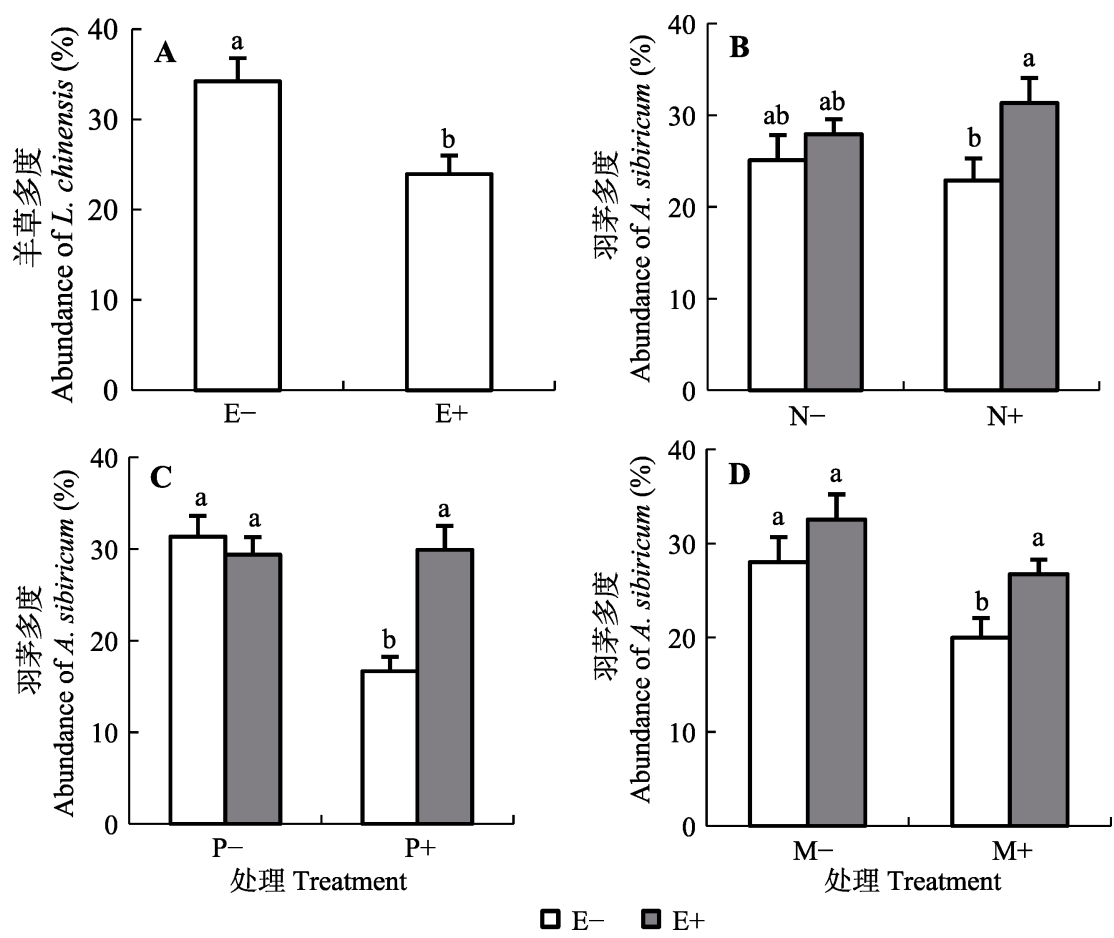

图4 不同处理条件下内生真菌感染对羊草 $(\mathbf{A})$ 和羽茅 $(\mathbf{B}-\mathbf{D})$ 多度的影响。 $\mathbf{B}$, 氮添加。C, 磷添加。D, 刏割处理(平均值+标准 误差, $n=5)$ ) E-, 无内生真菌感染; $\mathrm{E}+$, 内生真菌感染; $\mathrm{M}-$, 无刈割处理; $\mathrm{M}+$, 刈割处理; $\mathrm{N}-$, 无氮添加; $\mathrm{N}+$, 氮添加; $\mathrm{P}-$, 无磷 添加; $\mathrm{P}+$, 磷添加。不同小写字母表示 $\mathrm{E}+、 \mathrm{E}-$ 植物群落间差异显著 $(p<0.05)$ 。

Fig. 4 Effects of endophyte infection on the abundance of Leymus chinensis (A) and Achnatherum sibiricum (B-D). B, nitrogen addition. $\mathbf{C}$, phosphorus addition. D, mowing treatment (mean $+S E, n=5$ ). E-, endophyte-uninfected; E+, endophyte-infected; M-, no-mowing; $\mathrm{M}+$, mowing; $\mathrm{N}-$, no-nitrogen addition; $\mathrm{N}+$, nitrogen addition; $\mathrm{P}-$, no-phosphorus addition; $\mathrm{P}+$, phosphorus addition. Different lowercase letters denote significant difference between $\mathrm{E}+$ and $\mathrm{E}-$ plants $(p<0.05)$.
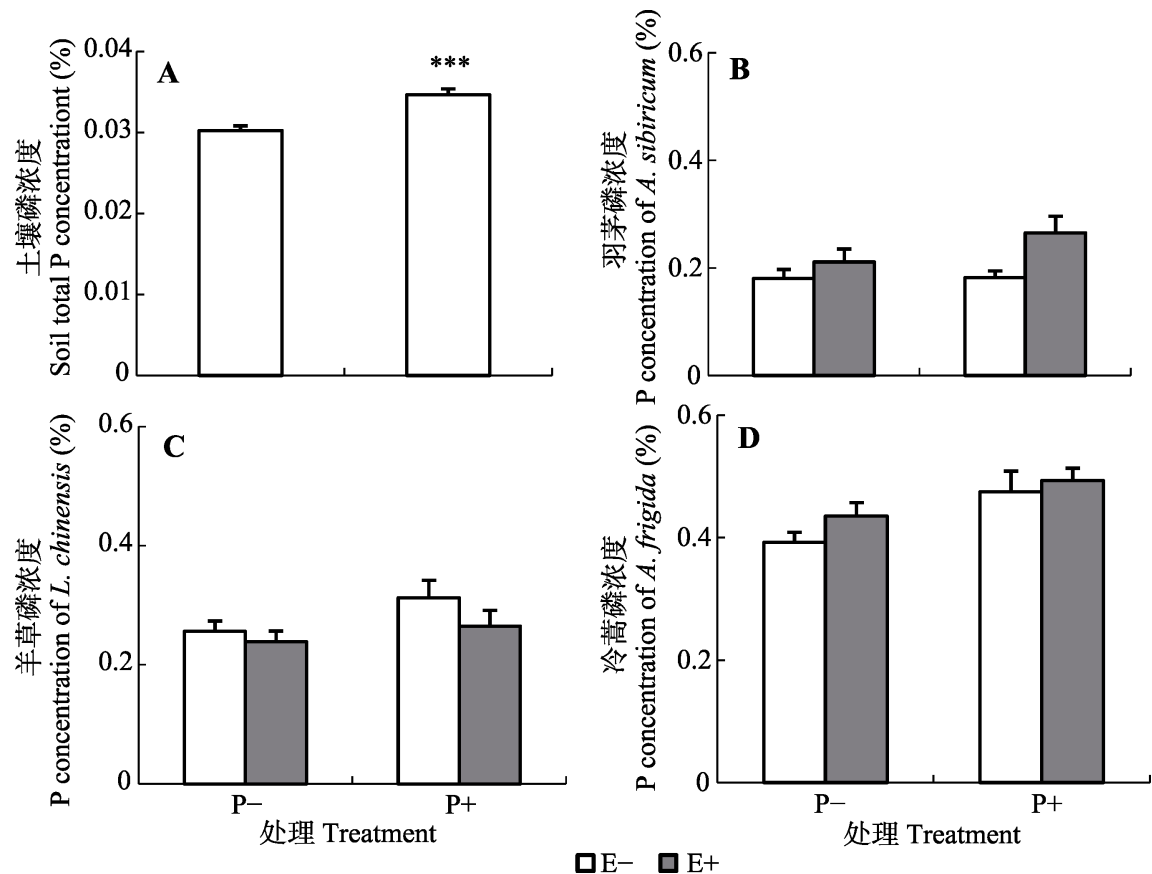

图5 磷添加对土壤 $(\mathbf{A})$ 和优势种 $(\mathbf{B}-\mathbf{D})$ 磷浓度的影响。 $\mathbf{B}$, 羽茅。 $\mathbf{C}$, 羊草。 $\mathbf{D}$, 冷蒿(平均值+标准误差, $n=5)$ - - -, 无内生真 菌感染; $\mathrm{E}+$, 内生真菌感染; $\mathrm{P}-$, 无磷添加; $\mathrm{P}+$, 磷添加。***, $p<0.001$ 。

Fig. 5 Effects of phosphorus addition on $\mathrm{P}$ concentration of the soil (A) and dominant plant species (B-D). B, Achnatherum sibiricum. C, Leymus chinensis. D, Artemisia frigida (mean $+S E, n=5$ ). E-, endophyte-uninfected; E+, endophyte-infected; P-, no-phosphorus addition; $\mathrm{P}+$, phosphorus addition. ${ }^{* *}, p<0.001$. 
et al., 1991; Rudgers \& Orr, 2009), 那么可能也会对 宿主所在群落的结构和多样性产生影响, 但到目前 为止, 相关的研究报道较少。Clay和Holah (1999)经 过连续4年的研究发现, 内生真菌感染显著提高了 宿主植物苇状羊茅的优势度, 降低了群落的物种多 样性, 但对群落生产力无显著影响。

Afkhami和Strauss (2016)对天然禾草雀麦属植 物为期 3 年的研究发现, 内生真菌能够增加非优势 种宿主植物的优势度, 降低优势种非宿主植物的优 势度, 从而使群落的多样性得到一定的提高。本研 究发现, 在刈割处理下, 内生真菌感染显著增加了 宿主植物羽茅以及多度较小的冰草和大针茅的多度, 而显著降低了优势种——羊草的多度, 使群落更加 均匀, 从而有利于提高群落多样性。

作为草地利用和管理的主要方式, 适度刈割可 能增加地表光可用性、降低物种间的竞争强度 (Grime，1973)，进而促进物种的共存(Cao et al., 2011)。但不同物种对刈割的响应不同, Knapp等 (2012)的研究表明禾本科植物因为刈割丢失的生物 量能够被那些快速生长、较矮的杂类草所补偿, 且 在底层植物较多的群落中这种现象尤为明显 (Bissels et al., 2004)。本研究结果表明: 内生真菌感 染对群落多样性的影响与刈割处理有关, 在刏割条 件下, 内生真菌感染显著增加了宿主植物羽茅以及 群落中多度较小的冰草和大针茅的多度, 而显著降 低了宿主植物羽茅所在群落中的优势种——羊草的 多度, 推测可能与内生真菌能够影响宿主植物的化 感作用有关, 而刈割处理进一步加大了这一影响。 已有研究表明, 内生真菌感染能够通过改变宿主植 物的化学组成而影响宿主植物的化感作用, 如 Sutherland等(1999)发现感染内生真菌的多年生黑麦 草对白三叶草(Trifolium repens)的化感效应显著强 于未染菌植物; Vázquez-de-Aldana等(2011)的研究 也表明相比于未染菌宿主植物, 染菌高羊茅的根部 浸提液能显著抑制百脉根(Lotus corniculatus) 和车 前草(Plantago lanceolata) 等目标植物的幼苗生长; 高远等(2017)研究表明, 感染内生真菌可缓解宿主 羽茅对大针茅的化感作用。另外, 有研究报道苇状 羊茅(Sullivan et al., 2007)和羽茅(Qin et al., 2016)受 到刚割干扰后, 染菌植株的生物碱等次生防御物质 的含量会有所增加, 考虑到不同物种对次生代谢物 的响应可能不同, 次生代谢物的增加可能抑制了优
势物种羊草的多度，而增加了大针茅等的多度，当 然, 起作用的化感物质的种类和含量还需进一步地 研究。

本研究结果与Afkhami和Strauss (2016)在天然 禾草中的研究结果相似, 而与Clay和Holah (1999)在 人工禾草中的研究结果不同, 其可能的原因包括: 在Clay和Holah (1999)研究的群落中宿主植物是人 工禾草苇状羊茅, Afkhami和Strauss (2016)研究的自 然群落宿主植物是天然禾草Bromus laevipes, 本实 验研究的宿主植物羽茅也是天然禾草。天然禾草内生真菌共生体与群落中其他物种之间的关系是在 生物和非生物的选择压力下经历漫长的演替过程而 形成的, 因此其对群落中其他物种的影响可能不同 于新引进的人工禾草-内生真菌共生体的影响。另一 方面, 在Clay和Holah (1999)研究的群落中宿主植物 苇状羊茅是群落优势种, Afkhami和Strauss (2016)研 究的群落中宿主植物B. laevipes 是非优势种。在前者 的群落中, 内生真菌因促进优势种的生长而降低了 群落多样性; 而在后者的群落中, 内生真菌因促进 非优势种的生长而增加了群落的多样性。而本研究 构建的人工群落, 在无刈割处理下羽茅是群落的优 势种, 而在刚割处理下不染菌羽茅成为非优势种, 我们同样发现内生真菌通过促进非优势种的生长而 增加了群落的多样性。在自然生境中, 羽茅常常作 为稳定的伴生种存在, 根据本研究结果我们推测: 羽茅的稳定存在与内生真菌感染有关, 并且随着刈 割作用, 内生真菌会通过促进宿主羽茅的生长而有 利于宿主所在群落多样性的维持, 当然推测还需进 一步的原位实验来验证。

越来越多的研究表明, 内生真菌对宿主植物的 影响与所在环境的养分供应有关。在氮供应方面, 目前的研究大多认为当宿主植株处于低氮生长环境 时, 内生真菌会因为对养分的消耗而对宿主产生不 利影响(Marks \& Clay, 2007), 但也有少数研究认为 内生真菌在低氮生长条件下对宿主有利(Arachavaleta et al., 1989; Cheplick et al., 1989; Lewis et al., 1996; Ravel et al., 1997; Newman et al., 2003; Lewis et al., 2004; Ren et al., 2009)。在磷供应方面, 以人工 禾草苇状羊茅和黑麦草为对象的研究表明, 在低磷 处理下, 内生真菌能够提高禾草宿主对土壤磷胁迫 的抵御能力, 而在正常供磷条件下, 内生真菌对宿 主的有益贡献消失(Malinowski et al., 1998, 1999, 
2000; Ren et al., 2007)。本研究表明, 内生真菌对宿 主羽茅的促进作用出现在供氮条件下，与已有的多 数报道相一致, 但内生真菌-禾草共生体对磷供应 的响应与已有的在人工禾草中的研究结果不同。已 有研究中, 只有在低磷条件下内生真菌感染才会促 进宿主植物的生长(Malinowski et al., 1999; 周芳等, 2003), 本研究中磷添加显著降低E-羽茅的多度, 但 对 $\mathrm{E}+$ 羽茅无显著影响, 导致在磷添加条件下内生真 菌感染显著提高了宿主植物的多度, 这可能与本实 验所采用的土壤本底中的养分含量有关。本实验所 用土壤是植物原生境土壤, 即使在无磷添加的处理 下, 土壤的全磷浓度仍达到 $0.03 \%$, 没有出现已报 道文献中的低磷处理。进一步对优势种磷浓度的分 析发现, E-羽茅所在群落中, 羽茅的磷浓度未随磷 添加而增加, 而羊草和冷蒿的磷浓度均有随磷添加 而增加的趋势(分别增加了 $22 \%$ 和 $21 \%$ ); 对于 $\mathrm{E}+$ 羽 茅群落, 群落中优势种的磷浓度均有随磷添加而增 加的趋势, 只是增加幅度不同, 所以我们推测: E羽茅群落中磷添加导致E-羽茅多度下降可能与磷 添加促进羊草和冷蒿等的生长而间接抑制了羽茅的 生长有关; 而对于 $\mathrm{E}+$ 羽茅群落, 磷添加对生长影响 的差异不明显, 因此对 $\mathrm{E}+$ 羽茅的多度影响不显著。

本研究发现氮磷添加对内生真菌作用的影响只 出现在宿主植物水平, 未对群落产生显著影响, 其 原因一方面可能与群落自身的组成有关, 在此群落 中羽茅、羊草、冷蒿多度接近, 羽茅并未占据绝对 优势地位, 所以羽茅对养分添加的响应没有在群落 多样性和生产力方面得到体现; 另一方面可能与不 同物种对养分添加的响应不同有关(Su et al., 2013), 本研究发现磷添加显著降低了群落中 E-羽茅的多 度, 却显著增加了群落中糙隐子草的多度, 因此对 总的生产力没有显著影响; 此外, 本实验只持续了 一个生长季, 而作为由多年生植物组成的群落而言, 可能有些差异还未得到充分体现。

\section{参考文献}

Afkhami ME, Strauss SY (2016). Native fungal endophytes suppress an exotic dominant and increase plant diversity over small and large spatial scales. Ecology, 97, 1159-1169.

Arachevaleta M, Bacon CW, Hoveland CS, Radcliffe DE (1989). Effect of the tall fescue endophyte on plant response to environmental stress. Agronomy Journal, 81, 83-90.

Arnold AE, Maynard Z, Gilbert GS, Coley PD, Kursar TA
(2000). Are tropical fungal endophytes hyperdiverse? Ecology Letters, 3, 267-274.

Bao SD (2000). Soil and Agricultural Chemistry Analysis. 3rd edn. China Agriculture Press, Beijing. 44-49. [鲍士旦 (2000). 土壤农化分析. 第三版. 中国农业出版社, 北 京. 44-49.]

Bissels S, Hölzel N, Otte A (2004). Population structure of the threatened perennial Serratula tinctoria in relation to vegetation and management. Applied Vegetation Science, 7, 267-274.

Brem D, Leuchtmann A (2002). Intraspecific competition of endophyte infected vs uninfected plants of two woodland grass species. Oikos, 96, 281-290.

Cao J, Holden NM, Lü XT, Du G (2011). The effect of grazing management on plant species richness on the QinghaiTibetan Plateau. Grass and Forage Science, 66, 333-336.

Cheplick GP (2004). Symbiotic fungi and clonal plant physiology. New Phytologist, 164, 413-415.

Cheplick GP, Clay K, Marks S (1989). Interactions between infection by endophytic fungi and nutrient limitation in the grasses Lolium perenne and Festuca arundinacea. New Phytologist, 111, 89-97.

Christensen MJ (1996). Antifungal activity in grasses infected with Acremonium and Epichloë endophytes. Australasian Plant Pathology, 25, 186-191.

Clay K (1987). Effects of fungal endophytes on the seed and seedling biology of Lolium perenne and Festuca arundinacea. Oecologia, 73, 358-362.

Clay K, Holah J (1999). Fungal endophyte symbiosis and plant diversity in successional fields. Science, 285, 1742-1744.

Collins CD, Foster BL (2009). Community-level consequences of mycorrhizae depend on phosphorus availability. Ecology, 90, 2567-2576.

Elmi AA, West CP (1995). Endophyte infection effects on stomatal conductance, osmotic adjustment and drought recovery of tall fescue. New Phytologist, 131, 61-67.

Faeth SH, Helander ML, Saikkonen KT (2004). Asexual Neotyphodium endophytes in a native grass reduce competitive abilities. Ecology Letters, 7, 304-313.

Gao Y, Li GP, Shi H, Liu H, Ren AZ, Gao YB (2017). Allelopathic effect of endophyte-infected Achnatherum sibiricum on Stipa grandis. Acta Ecologica Sinica, 37, 1063-1073. [高远, 李隔苹, 施宏, 刘慧, 任安芝, 高玉葆 (2017). 感染内生真菌的羽茅对大针茅的化感作用. 生态学报, 37, 1063-1073.]

Grime JP (1973). Competitive exclusion in herbaceous vegetation. Nature, 242, 344-347.

Janos DP (1980). Mycorrhizae influence tropical succession. Biotropica, 12, 56-64.

Jones TA, Gardner DR, Chatterton NJ (2000). Cattle prefer endophyte-free robust needle grass. Journal of Range Management, 53, 427-431.

Knapp AK, Hoover DL, Blair JM, Buis G, Burkepile DE, Chamberlain A, Collins SL, Fynn RWS, Kirkman KP, Smith MD, Blake D, Govender N, O’Neal P, Schreck T, 
Zinn A (2012). A test of two mechanisms proposed to optimize grassland aboveground primary productivity in response to grazing. Journal of Plant Ecology, 5, 357-365.

Lan ZC, Bai YF (2012). Testing mechanisms of N-enrichmentinduced species loss in a semiarid Inner Mongolia grassland: Critical thresholds and implications for long- term ecosystem responses. Philosophical Transactions of the Royal Society B, 367, 3125-3134.

Latch GCM, Christensen M (1985). Artificial infection of grasses with endophytes. Annals of Applied Biology, 107, $17-24$.

Leuchtmann A, Bacon CW, Schardl CL (2014). Nomenclatural realignment of Neotyphodium species with genus Epichloë. Mycologia, 106, 202-215.

Lewis GC (2004). Effects of biotic and abiotic stress on the growth of three genotypes of Lolium perenne with and without infection by the fungal endophyte Neotyphodium lolii. Journal of Chemical Ecology, 144, 53-63.

Lewis GC, Bakken AK, MacDuff JH, Raistrick N (1996). Effect of infection by the endophytic fungus Acremonium lolii on growth and nitrogen uptake by perennial ryegrass (Lolium perenne) in flowing solution culture. Annals of Applied Biology, 129, 451-460.

Li CJ, Nan ZB, Paul VH, Dapprich PD, Liu Y (2004). A new Neotyphodium species symbiotic with drunken horse grass (Achnatherum inebrians) in China. Mycotaxon, 90, 141-147.

Li X, Han R, Ren AZ, Gao YB (2010). Using high-temperature treatment to construct endophyte-free Achnatherum sibiricum. Microbiology China, 37, 1395-1400. [李夏, 韩 荣, 任安芝, 高玉葆 (2010), 高温处理构建不感染内生 真菌羽茅种群的方法探讨. 微生物学通报, 37, 1395-1400.]

Li XS, Zhang XZ, Gu X, Zheng Y (1998). The relationship between the toxic metabolin and the endophytic fungi of drunken horse grass (Achnatherum inebrians). GrassFeeding Livestock, 124, 44-46. [李学森, 张学洲, 顾祥, 郑瑛 (1998). 醉马草有毒物质与其内生真菌的关系. 草 食家畜, 124, 44-46.]

Louis MS, Faeth SH (1997). The effect of endophytic fungi on the fitness of Arizona fescue (Festuca arizonica) under varying nitrogen levels. Bulletin of the Ecological Society of America, 78, 317-327.

Malinowski DP, Alloush GA, Belesky DP (2000). Leaf endophyte Neotyphodium coenophialum modifies mineral uptake in tall fescue. Plant and Soil, 227, 115-126.

Malinowski DP, Belesky DP (2006). Ecological importance of Neotyphodium spp. grass endophytes in agroecosystems. Grassland Science, 52, 1-14.

Malinowski DP, Belesky DP, Hill NS, Baligar VC, Fedders JM (1998). Influence of phosphorus on the growth and ergot alkaloid content of Neotyphodium coenophialum-infected tall fescue (Festuca arundinacea Schreb.). Plant and Soil, 198, 53-61.

Malinowski DP, Brauer DK, Belesky DP (1999). The endo- phyte Neotyphodium coenophialum affects root morphology of tall fescue grown under phosphorus deficiency. Journal of Agronomy and Crop Science, 183, 53-60.

Malinowski DP, Leuchtmann A, Schmidt D, Nosberger J (1997). Symbiosis with Neotyphodium uncinatum endophyte may increase the competitive ability of meadow fescue. Agronomy Journal, 89, 833-839.

Marks S, Clay K (2007). Low resource availability differentially affects the growth of host grasses infected by fungal endophytes. International Journal of Plant Sciences, 168, 1269-1277.

Marks S, Clay K, Cheplick GP (1991). Effects of fungal endophytes on interspecific and intraspecific competition in the grasses Festuca arundinacea and Lolium perenne. Journal of Applied Ecology, 28, 194-204.

Moraes PVD, Witt WW, Phillips TD, Rossi P, Panozzo LE (2014). Relative competitiveness of Nimblewill (Muhlenbergia schreberi) with tall fescues and kentucky bluegrass. American Journal of Plant Sciences, 5, 3777-3787.

Newman JA, Abner ML, Dado RG, Gibson DJ, Brookings A, Parson AJ (2003). Effects of elevated $\mathrm{CO}_{2}$, nitrogen and fungal endophyte-infection on tall fescue: Growth, photosynthesis, chemical composition and digestibility. Global Change Biology, 9, 425-437.

Oberhofer M, Gusewell S, Leuchtmann A (2014). Effects of natural hybrid and non-hybrid Epichloë endophytes on the response of Hordelymus europaeus to drought stress. New Phytologist, 201, 242-253.

Qin JH, Gao Y, Liu H, Zhou Y, Ren AZ, Gao YB (2016). Effect of Endophyte infection and clipping treatment on resistance and tolerance of Achnatherum sibiricum. Frontiers in Microbiology, 7, 1988. DOI: 10.3389/fmicb.2016.01988.

Ravel C, Courty C, Coudret A, Charmet G (1997). Beneficial effects of Neotyphodium lolii on the growth and the water status in perennial ryegrass cultivated under nitrogen deficiency or drought stress. Agronomie, 17, 173-181.

Ren AZ, Gao YB, Wang W, Wang JL, Zhao NX (2009). Influence of nitrogen fertilizer and endophyte infection on ecophysiological parameters and mineral element content of perennial ryegrass. Journal of Integrative Plant Biology, $51,75-83$.

Ren AZ, Gao YB, Zhou F (2007). Response of Neotyphodium lolii-infected perennial ryegrass to phosphorus deficiency. Plant Soil and Environment, 53, 113-119.

Rudgers JA, Clay K (2008). An invasive plant-fungal mutualism reduces arthropod diversity. Ecology Letters, 11, 831-840.

Rudgers JA, Orr S (2009). Non-native grass alters growth of native tree species via leaf and soil microbes. Journal of Ecology, 97, 247-255.

Saikkonen K, Lehtonen P, Helander M, Koricheva J, Faeth SH (2006). Model systems in ecology: Dissecting the endophytegrass literature. Trends in Plant Science, 11, 428-433.

Schardl CL, Young CA, Faulkner JR, Florea S, Pan J (2012). Chemotypic diversity of Epichloë, fungal symbionts of

www.plant-ecology.com 
grasses. Fungal Ecology, 5, 331-344.

Schulthess FM, Faeth SH (1998). Distribution, abundances, and associations of the endophytic fungal community of Arizona fescue (Festuca arizonica). Mycologia, 90, 569-578.

Su JQ, Li XR, Li XJ, Li F (2013). Effects of additional N on herbaceous species of desertified steppe in arid regions of China: A four-year field study. Ecological Research, 28, 21-28.

Sullivan TJ, Rodstrom J, Vandop J, Librizzi J, Graham C, Schardl CL, Bultman TL (2007). Symbiont-mediated changes in Lolium arundinaceum inducible defenses: Evidence from changes in gene expression and leaf composition. New Phytologist, 176, 673-679.

Sutherland BL, Hume DE, Tapper BA (1999). Allelopathic effects of endophyte-infected perennial ryegrass extracts on white clover seedlings. New Zealand Journal of Agricultural Research, 42, 19-26.

Vázquez-de-Aldana BR, Romo M, García-Ciudad A, Petisco C, García-Criado B (2011). Infection with the fungal endophyte Epichloe festucae may alter the allelopathic potential of red fescue. Annals of Applied Biology, 159, 281-290.

Vázquez-de-Aldana BR, Zabalgogeazcoa I, García-Ciudad A, García- Criado B (2013). An Epichloë endophyte affects the competitive ability of Festuca rubra against other grassland species. Plant \& Soil, 362, 201-213.

Wei YK, Gao YB, Xu H, Su D, Zhang X, Wang YH, Lin F, Chen L, Nie LY, Ren AZ (2006). Occurrence of endophytes in grasses native to northern China. Grass and Forage Science, 61, 422-429.

White JF, Reddy PV (1998). Examination of structure and mo- lecular phylogenetic relationships of some graminicolous symbionts in genera Epichloë and ParEpichloë. Mycologia, 90, 226-234.

Xia C, Zhang XX, Christensen MJ, Nan ZB, Li CJ (2015). Epichloe endophyte affects the ability of powdery mildew (Blumeria graminis) to colonise drunken horse grass (Achnatherum inebrians). Fungal Ecology, 16, 26-33.

Yang GW, Liu N, Lu WJ, Wang S, Kan HM, Zhang YJ, Xu L, Chen YL (2014). The interaction between arbuscular mycorrhizal fungi and soil phosphorus availability influences plant community productivity and ecosystem stability. Journal of Ecology, 102, 1072-1082.

Yang GW, Yang X, Zhang WJ, Wei YQ, Ge G, Lu WJ, Sun JJ, Liu N, Kan HM, Shen Y, Zhang YJ (2015). Arbuscular mycorrhizal fungi affect plant community structure under various nutrient conditions and stabilize the community productivity. Oikos, 125, 576-585.

Zhang X, Ren AZ, Wei YK, Lin F, Li C, Liu ZJ, Gao YB (2009). Taxonomy, diversity and origins of symbiotic endophytes of Achnatherum sibiricum in the Inner Mongolia Steppe of China. FEMS Microbiology Letters, 301, 12-20.

Zhou F, Gao YB, Ma WJ (2003). Effects of phosphorus deficiency on growth of perennial ryegrass-fungal endophyte symbiont and phenolic content in root. Plant Physiology Communications, 39，321-324. [周芳, 高玉葆, 马文江 (2003). 缺磷对黑麦草-内生真菌共生体生长和根中酚 含量的影响. 植物生理学通讯, 39, 321-324.]

责任编委：陈保冬 责任编辑: 李 敏

附录I 在不同刈割条件下内生真菌感染和氮、磷添加对群落多样性影响的三因素方差分析

Supplement I Three-way ANOVA of the effects of endophyte infection, nitrogen and phosphorus addition on community diversity under different mowing levels

http://www.plant-ecology.com/fileup/PDF/cjpe.2018.0242-S1.PDF

附录II 内生真菌感染、养分添加和刈割处理对群落生产力影响的多因素方差分析

Supplement II Multiple ANOVA of the effects of endophyte infection, nitrogen and phosphorus addition, and mowing on the community productivity

http://www.plant-ecology.com/fileup/PDF/cjpe.2018.0242-S2.PDF

附录III 在不同刈割条件下内生真菌感染和养分作用对群落物种多度影响的三因素方差分析

Supplement III Three-way ANOVA of the effects of endophyte infection and nutrients addition on species abundance under non-mowing or mowing conditions

http://www.plant-ecology.com/fileup/PDF/cjpe.2018.0242-S3.PDF

附录IV 内生真菌感染和养分添加以及刈割作用对优势植物多度影响的多因素方差分析

Supplement IV Multiple ANOVA the effects of endophyte infection, nutrients addition and mowing on the abundance of dominant species

http://www.plant-ecology.com/fileup/PDF/cjpe.2018.0242-S4.PDF

附录 $\mathbf{V}$ 内生真菌感染、养分添加和划割处理对土壤、羽茅、羊草和冷蒿磷浓度的多因素方差分析

Supplement $V$ Multiple ANOVA of effects of endophyte infection, nutrients addition and mowing on $P$ concentration of the soil, Achnatherum sibiricum, Leymus chinensis and Artemisia frigida

http://www.plant-ecology.com/fileup/PDF/cjpe.2018.0242-S5.PDF 